\title{
Anticiper l'avenir des territoires agricoles en Afrique de l'Ouest: le cas des Niayes au Sénégal
}

\author{
Clémentine Camara ${ }^{1, *}$, Robin Bourgeois ${ }^{2,3,4}$ et Camille Jahel ${ }^{5,6,7}$ \\ ${ }^{1}$ Montpellier SupAgro, Institut des régions chaudes, 1101, avenue Agropolis, BP 5098, 34093 Montpellier cedex 05, France \\ 2 CIRAD, UMR ART-DEV, 34398 Montpellier, France \\ 3 ART-DEV, Univ Montpellier, Montpellier, France \\ ${ }^{4}$ Centre for the Study of Governance Innovation, Old College House, University of Pretoria, Hatfield Campus, Lynnwood Road, \\ 0002 Pretoria, Afrique du Sud \\ ${ }^{5}$ CIRAD, UMR TETIS, 34398 Montpellier, France \\ 6 TETIS, Univ Montpellier, Montpellier, France \\ ${ }^{7}$ ISRA BAME, route des hydrocarbures, BP 3120, Dakar, Sénégal
}

\begin{abstract}
Résumé - En Afrique de l'Ouest, dans le contexte général de changement climatique et de mondialisation, la croissance démographique exerce une pression accrue sur les ressources naturelles par les changements d'affectation des sols qu'elle engendre. L'avenir des territoires agricoles, en particulier en périphérie des grandes villes, est alors questionné face à l'urbanisation croissante, la dégradation des ressources naturelles et les mutations socio-économiques. Afin d'anticiper les changements que pourraient connaitre les territoires ruraux, une démarche de prospective territoriale a été réalisée sur la zone sud des Niayes, au Sénégal. Elle a permis aux experts locaux mobilisés à cet effet d'identifier les facteurs de changement et de co-élaborer des scénarios d'évolutions plausibles du territoire. Les réglementations, la gouvernance et la démographie sont les trois facteurs majeurs pouvant infléchir de l'affectation des sols dans le temps. Ces facteurs déterminent le type de partage de l'espace et d'utilisation des ressources naturelles, pouvant être harmonieux ou conflictuel. Une gouvernance inclusive, une société civile fortement structurée et la préservation des ressources naturelles sont apparues comme essentielles à l'atteinte de futurs harmonieux.
\end{abstract}

Mots clés : prospective territoriale / anticipation / territoires ruraux / urbanisation / Niayes

\begin{abstract}
Anticipating the future of agricultural territories in West Africa: The case of the Niayes in Senegal. In West Africa, in the global context of climate change and globalization, population growth creates an increasing pressure on natural resources through land allocation changes that it generates. The future of agricultural territories, especially around large cities, is thus questioned considering increasing urbanization, degradation of natural resources and socio-economic transformations. In order to anticipate what changes rural areas could experience, a process of territorial foresight was conducted on the southern Niayes area in Senegal. It enabled the local experts who engaged in this process to identify factors of change and to co-elaborate plausible scenarios of change in the territory. Regulations, governance and demography are the three major factors affecting land allocation over time. These factors determine how space is shared and natural resources are used, harmoniously or through conflict. Inclusive governance, highly structured civil society, but also the preservation of natural resources have emerged as essential to achieving harmonious futures.
\end{abstract}

Keywords: territorial foresight / anticipation / rural territory / urbanization / Niayes

\section{Introduction}

Dans les pays d'Afrique de l'Ouest, la rapidité et l'ampleur de la croissance démographique engendrent des taux d'urbanisation sans précédent (Halleux, 2015). En outre, la

\footnotetext{
*Auteur de correspondance : clementine.camara@gmail.com
}

conjonction d'aléas climatiques, de processus de dégradation des ressources naturelles, ainsi que la diminution des terres cultivables disponibles par habitant, induisent depuis plusieurs décennies des migrations de populations qui tendent à se concentrer dans les zones encore fertiles, mais aussi et surtout dans les villes et à leurs périphéries (Alex et Gemenne, 2016; Mercandalli et Losch, 2018). Ainsi, les espaces agricoles péri- 


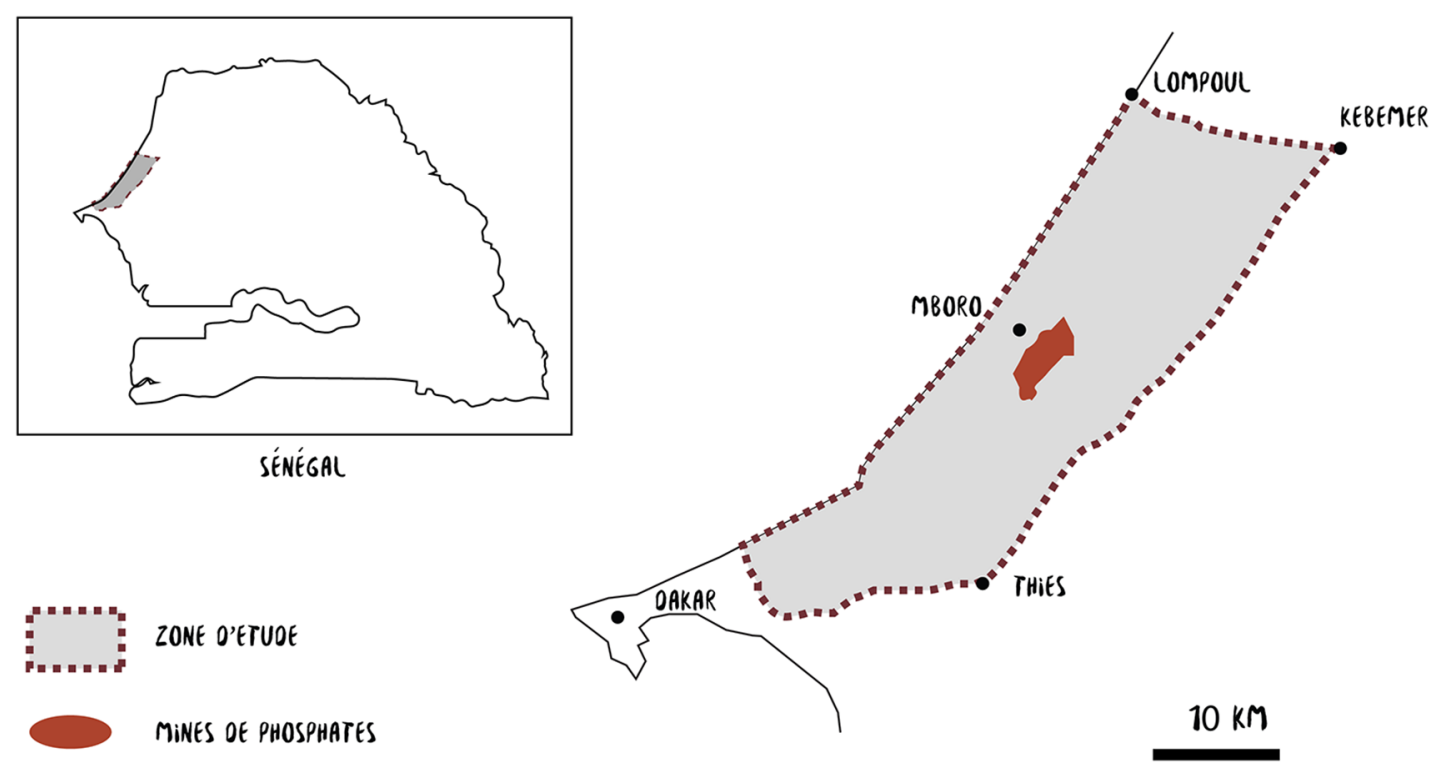

Fig. 1. Carte de la zone des Niayes et délimitation de la zone d'étude (source: auteurs).

Fig. 1. Map of the Niayes area and delimitation of the study area (source: authors).

urbains sont confrontés à l'enjeu majeur de contribuer à nourrir une population croissante dans un contexte de dégradation et de raréfaction des ressources naturelles (Bispo et al., 2017; Foley et al., 2005) mais aussi d'urbanisation. Suivant la politique d'aménagement adoptée et les relations entre acteurs (Robineau et Dugué, 2018), les villes peuvent alors être considérées soit comme un moteur de développement en offrant des ressources et des opportunités de marché aux agriculteurs (Bricas et Seck, 2004), soit comme des prédatrices de l'espace rural, diminuant l'espace alloué aux activités productives et générant de nombreuses pollutions qui peuvent dégrader la qualité des aliments produits (Temple et Moustier, 2004).

Sous l'effet conjugué d'un grand nombre de facteurs de changement, les territoires et leurs formes d'organisations sociales évoluent en permanence; l'avenir de ces espaces et de leurs habitants est de ce fait incertain. Il est cependant possible de mobiliser des approches permettant d'anticiper les changements susceptibles d'affecter le cadre de vie des populations ou la capacité productive des territoires agricoles. L'analyse prospective à l'échelle d'un territoire permet de tendre vers cette voie (Bourgeois et al., 2017).

La prospective territoriale est un outil d'anticipation qui consiste à explorer des scénarios plausibles d'évolution d'un territoire. En France, 1'application de démarches de prospective à l'échelle des territoires est ancrée dans les travaux de la Délégation interministérielle à l'aménagement du territoire et à l'attractivité régionale (DATAR) (Mirenowicz, 1991); sa pratique a longtemps été comprise comme une démarche en appui à l'action publique et visant à l'aménagement de territoires (Loinger et Spohr, 2005). En ce sens, elle est réalisée à la demande d'un commanditaire public (État ou collectivité locale), avec un cahier des charges et des processus dérivés de la prospective d'entreprise (Spohr, 2009). Pour les géographes, elle apparaît comme un outil de l'ingénierie de construction des territoires (Fourny et Denizot, 2007) reposant sur une dimension spatialisée liée à l'exploration du futur (Lardon et Noucher, 2016). Avec le développement de démarches dites participatives, la pratique de l'anticipation à l'échelle territoriale est devenue plus inclusive et moins dépendante de la volonté des pouvoirs publics.

En ce sens, le travail présenté ici se caractérise par sa distance avec une intervention publique d'aménagement, puisqu'il trouve son origine dans une initiative de chercheurs qui a pu mobiliser des acteurs locaux autour d'une réflexion endogène sur la transformation de l'espace agro-sylvo-pastoral de la zone sud des Niayes. Par ailleurs, il est original dans le fait qu'il vise à faire sens du présent avant de cibler des actions de nature plus stratégique, suivant en cela une pratique de l'anticipation visant aussi à produire une culture du futur (Miller, 2018).

L'objectif de cet article est de présenter et de discuter une démarche participative de co-élaboration de scénarios pour explorer une diversité de futurs plausibles d'un territoire soumis à de fortes pressions anthropiques et climatiques, et d'examiner les points d'inflexion de ces différentes trajectoires d'évolution. Ce territoire d'étude est la zone des Niayes, au Sénégal.

\section{Méthodologie et données}

\subsection{Zone d'étude}

La zone d'étude se situe dans la partie sud des Niayes, bande côtière entre Dakar et Saint-Louis (Fig. 1), et couvre une superficie d'environ $2300 \mathrm{~km}^{2}$.

De par les potentialités de ses sols, son climat tropical atténué par les alizés maritimes et l'existence de ressources hydriques conséquentes et à faible profondeur, c'est une zone agricole importante, concentrant la majorité de la production horticole du Sénégal (Fare et al., 2017). Mais la richesse de son sous-sol en fait aussi une zone d'extraction minière privilégiée, 
Tableau 1. Les étapes de la co-élaboration de scénarios.

Table 1. Steps of the scenario co-building process.

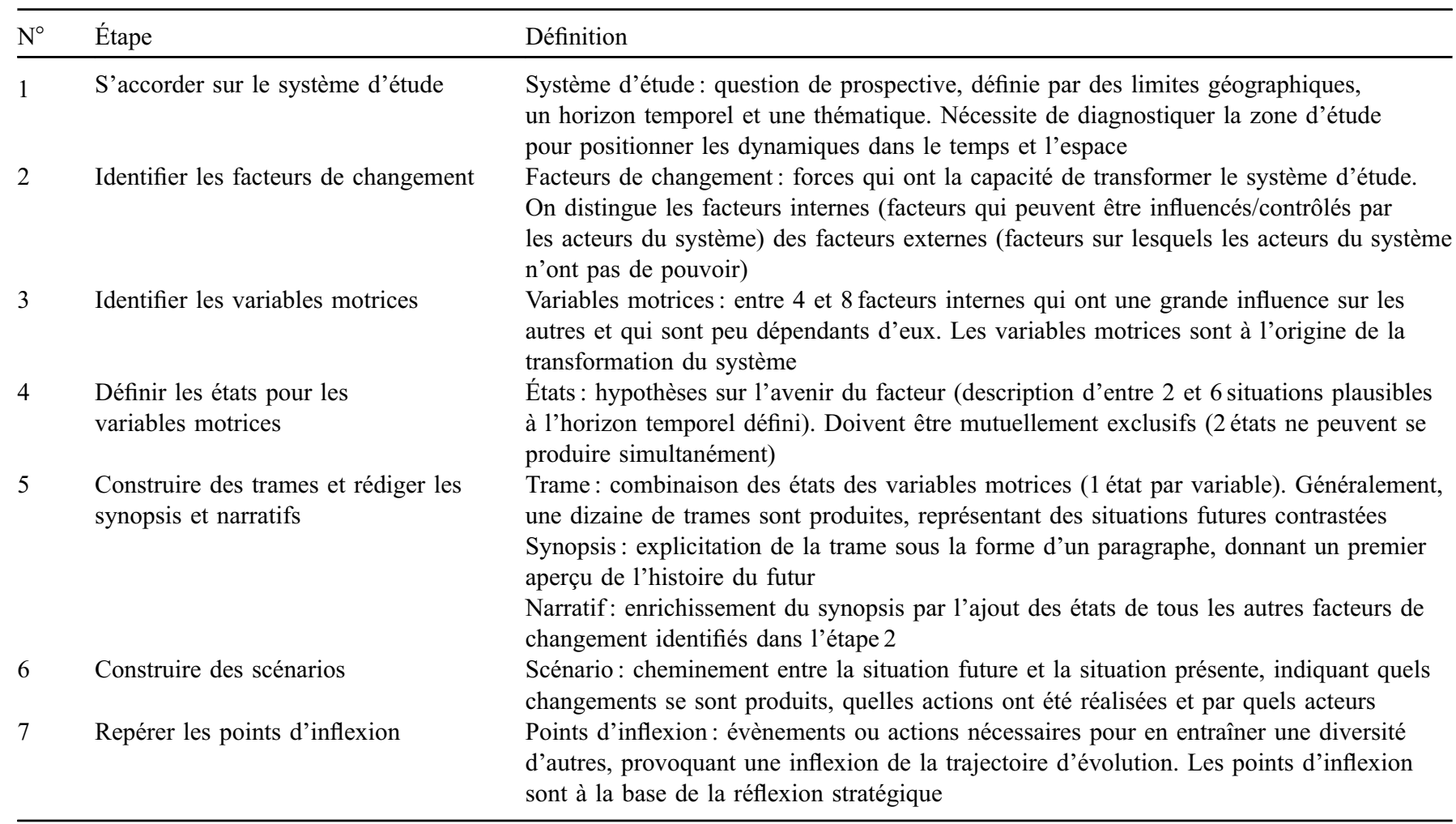

notamment pour le phosphate et le zircon (CEDEAO, 2010; Fall et Fall, 2001).

Aujourd'hui, les territoires agricoles de la zone sud des Niayes sont menacés par l'émergence de nouvelles dynamiques. L'urbanisation et les projets étatiques - notamment l'autoroute, l'aéroport international et la nouvelle ville de Diamniado - empiètent rapidement sur les terres agricoles (Wade, 2015). L'essor d'unités de production agricole et de transformation capitalistes, ainsi que des activités minières, fortement soutenues par les politiques publiques, questionne l'avenir de l'agriculture familiale de la zone (Bosc et Losch, 2002 ; FAO, 2017). Enfin, la baisse du niveau de la nappe et sa salinisation progressive, suite aux sécheresses et à une augmentation de son utilisation, menacent toute la production maraîchère (DGPRE, 2014; Fall et Fall, 2001).

On observe donc une tension entre les différentes occupations des sols (agricoles, industrielles, infrastructures ou résidentielles) et une concurrence forte entre différentes formes d'agriculture, entraînant une compétition pour l'accès aux différentes ressources, notamment le foncier.

\subsection{Méthode utilisée pour la construction des scénarios}

Les ateliers de co-élaboration des scénarios exploratoires d'évolution du territoire ont été réalisés à partir de la méthodologie développée au CIRAD par Bourgeois et al. (2017).

La co-élaboration des scénarios fait intervenir un groupe «d'experts locaux », personnes sélectionnées pour leurs connaissances du terrain et leurs compétences, et non pour représenter une organisation ou des groupes d'intérêt. Les scénarios étant des images complexes et plausibles du futur d'un territoire réel, leur élaboration nécessite de nombreuses connaissances spécifiques et complémentaires afin que toutes les composantes socio-économiques et biophysiques d'un territoire soient couvertes. Les compétences des 21 experts locaux (agents d'organisations paysannes, du gouvernement, de l'université, salariés de bureaux d'études, etc.), identifiés avec l'aide d'un comité de pilotage composé de trois organisations d'appui aux dynamiques paysannes, couvraient donc un ensemble de dix dimensions retenues lors d'une étape préliminaire de diagnostic territorial pour décrire le territoire dans sa globalité: le milieu naturel, l'histoire et l'organisation sociale de la zone, les tendances démographiques et migratoires, l'économie locale (agriculture, élevage, commerce, industrie, tourisme, etc.), l'aménagement du territoire et l'accès au foncier, les réseaux de communication, l'assainissement et le traitement des déchets, l'accès à la santé et à la formation (Camara et al., 2018).

La co-élaboration de scénarios a d'abord consisté à identifier une liste de facteurs de changements. Une discussion sur leur influence et leur dépendance mutuelle a ensuite permis de déterminer parmi ces facteurs un nombre restreint de « variables motrices » dans l'évolution future du territoire. Des hypothèses sur l'état du futur de chacune de ces variables motrices ont été produites par les experts au cours d'un travail de réflexion collective. Différentes images du futur ont été créées en combinant de manière cohérente ces différentes hypothèses. Les scénarios complets ont été obtenus en imaginant les chemins d'action entre les images du futur et la situation présente (tableau 1) (Camara et al., 2018). 
Tableau 2. Identification et définition des variables motrices.

Table 2. Identification and definition of the driving forces.

\begin{tabular}{ll}
\hline Nom de la variable motrice & Définition de la variable motrice \\
\hline $\begin{array}{l}\text { Pression démographique } \\
\text { Infrastructures d'éducation et de formation }\end{array}$ & $\begin{array}{l}\text { Évolution et niveau de la pression démographique } \\
\text { État, couverture et accessibilité des infrastructures d'éducation et de formation } \\
\text { (capacité, nombre et emplacement) }\end{array}$ \\
$\begin{array}{l}\text { Eessource en eau } \\
\text { Systèmes de production agricole }\end{array}$ & $\begin{array}{l}\text { Type de système de production agricole (niveau de modernisation des exploitations } \\
\text { agricoles, types de technique d'irrigation et de fertilisation, techniques et technologies } \\
\text { employées, types de partenariats, localisation et emprise) et état de la production agricole } \\
\text { (type, qualité et quantité de produits dans la zone) }\end{array}$ \\
$\begin{array}{l}\text { Niveau de la qualité et de la fertilité des sols } \\
\text { Ressource sols }\end{array}$ & $\begin{array}{l}\text { État de la réglementation et de l'utilisation des sols } \\
\text { État de la réglementation et de l'utilisation } \\
\text { des sols }\end{array}$ \\
Gouvernance & Qui prend les décisions et comment elles sont prises \\
\hline
\end{tabular}

Tableau 3. Exemple des états futurs d'une variable motrice.

Table 3. Example of the future states for one driving force.

\begin{tabular}{|c|c|c|c|c|c|}
\hline $\begin{array}{l}\text { Numérotation } \\
\text { des états futurs }\end{array}$ & 1 & 2 & 3 & 4 & 5 \\
\hline $\begin{array}{l}\text { D. Systèmes } \\
\text { de production } \\
\text { agricole }\end{array}$ & $\begin{array}{l}\text { Exploitations familiales } \\
\text { qui optimisent les } \\
\text { facteurs de production } \\
\text { pour des produits } \\
\text { de qualité, orientés vers } \\
\text { le marché local et les } \\
\text { exportations, en partenariat } \\
\text { avec les agro-industries } \\
\text { et entreprises } \\
\text { de transformation }\end{array}$ & $\begin{array}{l}\text { Exploitations familiales } \\
\text { peu compétitives en } \\
\text { concurrence avec les } \\
\text { agro-industries sur les } \\
\text { marchés nationaux et } \\
\text { internationaux }\end{array}$ & $\begin{array}{l}\text { Disparition des exploitations } \\
\text { familiales au profit des } \\
\text { agro-industries non locales qui } \\
\text { approvisionnent le } \\
\text { marché national et international } \\
\text { en produit agricoles }\end{array}$ & $\begin{array}{l}\text { Disparition de } \\
\text { l'agriculture }\end{array}$ & $\begin{array}{l}\text { Micro-agriculture } \\
\text { entrepreneuriale } \\
\text { entièrement } \\
\text { robotisée }\end{array}$ \\
\hline
\end{tabular}

\section{Résultats}

\subsection{Les éléments constitutifs des scénarios}

Le groupe d'experts s'est accordé sur la question: «Quel avenir pour l'espace agro-sylvo-pastoral de la zone sud des Niayes à l'horizon 2040?» La zone d'étude a été définie comme indiqué dans la figure 1 .

Dix facteurs de changement externes et 43 facteurs de changement internes ont été identifiés (Camara et al., 2018). Parmi les facteurs de changement internes, sept variables motrices ont été sélectionnées par les experts, qui leur ont donné des définitions précises (tableau 2).

\subsection{Les scénarios d'évolutions plausibles}

Un exemple des états futurs d'une variable motrice est présenté dans le tableau 3, l'intégralité se trouvant dans le document de référence produit à la suite des ateliers (Camara et al., 2018). Leur combinaison a permis de co-élaborer neuf futurs plausibles à l'horizon 2040 (fig. 2).

Ces scénarios diffèrent par le type d'occupation de l'espace et par les modalités de coexistence entre les acteurs du territoire (fig. 3). Par exemple, les scénarios 5 et 9 présentent tous les deux une zone sud des Niayes totalement urbanisée, mais cette urbanisation conduit à une situation conflictuelle dans le premier, alors que les acteurs vivent en harmonie dans le second.

L'avenir de la zone sud des Niayes est donc pluriel et incertain, une multitude de futurs contrastés pouvant être envisagés. Quels évènements peuvent alors connecter le présent vers un scénario plutôt qu'un autre ? Quels acteurs ont un rôle à jouer et par quelles actions?

\subsection{Les points d'inflexion}

La construction des cheminements (Camara et al., 2018) a mis en évidence l'existence de protagonistes et d'actions particulières, appelées points d'inflexion (tableau 1), menant à un partage de l'espace harmonieux, conflictuel ou singulier (fig. 3).

Pour tous les scénarios, les réglementations - de la gestion des ressources naturelles, des flux migratoires, du foncier ou de l'utilisation des intrants par exemple - apparaissent comme la pierre angulaire des trajectoires d'évolution. Leur présence ou leur absence a en effet été chaque fois identifiée comme un 
1 L'Eldorado du Sénégal : Ville cosmopolite (expansion limitée à $20 \%$ du territoire), cohabitant harmonieusement avec les territoires agricoles. Réglementation équitable et respectée. Gouvernance inclusive (consultation). Emplois, très diversifiés, développés autour de la préservation de l'environnement.

2 Perfeco : Eco-villages, où l'agriculture est intégrée. Réglementation équitable et respectée. Gouvernance inclusive (concertation, aucune prise de décision sans accord préalable des habitants). Emplois, majoritairement liés à l'agriculture, développés autour de la préservation de l'environnement.

Robotisation de $\because \rightarrow 3$ Les éco-villages

l'agriculture $\rightarrow \rightarrow$ (3) deviennent des villes 4 (4) Ouvertur

5 La ville verte autogérée : Une seule ville s'étend sur toute la superficie. Populations s'autogèrent et mettent en place leurs propres systèmes de réglementations. Emplois très diversifiés. Agriculture robotisée hors-sol. Accès inégal aux services.

6 Les Niayes touristiques : Réserve touristique règlementée par l'Etat sur accord des populations. Emplois uniquement liés au tourisme (préservation de l'environnement).

Zone minée : Réserve pour l'extraction minière, détenue exclusivement par un conglomérat privé, qui a négocié les droits avec l'Etat (décision contestée par les populations).

SOS Niayes : Progression de l'étalement urbain, de manière chaotique (réglementation non respectée). Développement des industries minières et des agro-industries. Prise de pouvoir par des groupes privés. Délabrement de toutes les infrastructures.

II Niayes plus rien : Disparition de Dakar sous les eaux, nouveaux pôles urbains érigés de manière anarchique (réglementation non respectée). Développement de l'industrie minière et de trafics illicites (disparition de l'agriculture) par une mafia qui détient le pouvoir.

Fig. 2. Les neuf scénarios pour la zone sud des Niayes (source: auteurs).

Fig. 2. The nine scenarios for the southern Niayes area (source: authors).

point d'inflexion par les experts locaux. La nature des acteurs au pouvoir, de l'influence des autres acteurs sur la prise de décision finale et ainsi des modalités de cette prise de décision est également prégnante dans les trajectoires. L'avenir de l'espace étudié semble donc être intimement lié au type de gouvernance du territoire et aux outils mobilisés pour mettre en œuvre la vision portée par l'entité au pouvoir. En outre, plusieurs références au nombre d'habitants et à leur répartition dans l'espace ont été relevées; le seuil de pression démographique serait donc aussi un point d'inflexion important. Un exemple de cheminement, où apparaissent les points d'inflexion, est présenté en figure 4, l'intégralité des cheminements se trouvant dans le document de référence (Camara et al., 2018).

Trois principaux points d'inflexion émergent donc : la mise en place de réglementations, les modalités de gouvernance et la pression démographique. Il est à noter que tous les scénarios, sauf le premier, envisagent une situation conflictuelle avec une dégradation des ressources naturelles au début des trajectoires d'évolution.

\section{Discussion}

\subsection{Vers quel futur?}

Le scénario 8 est le seul dont l'avènement ne demanderait aucune action particulière; on y trouve plutôt une absence d'action. Il s'agirait donc d'un scénario tendanciel, qui décrit ce qui se passerait si les dynamiques passées et actuelles se poursuivaient. Le caractère alarmant de ce scénario pointe de fait l'urgence et la nécessité d'agir au présent. La question qui se pose alors est : comment dévier d'une trajectoire qui semble mener à une situation toujours plus chaotique?

Dans tous les scénarios à l'exception du scénario 1 , la dégradation des différentes ressources du territoire et les conflits pour y accéder jouent un rôle important (foncier, eau agricole et potable, etc.). Dans cinq scénarios $(2,3,4,5$ et 6$)$, la dégradation initiale de la situation agit en effet comme un électrochoc sur les populations et conduit ainsi à une certaine prise de conscience et à une reprise en main de l'avenir du territoire à travers l'implication de ses populations dans la prise de décision. Par exemple, le scénario 6 part d'une surexploitation des forages, une perte de certains sols, une épidémie et des conflits avant la mise en place de concertations. Inversement, dans trois autres scénarios (7, 8 et 9), les conflits empirent et génèrent des situations de plus en plus chaotiques.

Par ailleurs, pour tendre vers les scénarios harmonieux (scénarios 1, 2, 3 et 4), les experts ont donné une grande importance à l'implication d'un État fort, disposant des moyens nécessaires pour contrôler et sanctionner, agissant en entente avec une société civile et des organisations professionnelles fortement structurées. L'existence et le respect 


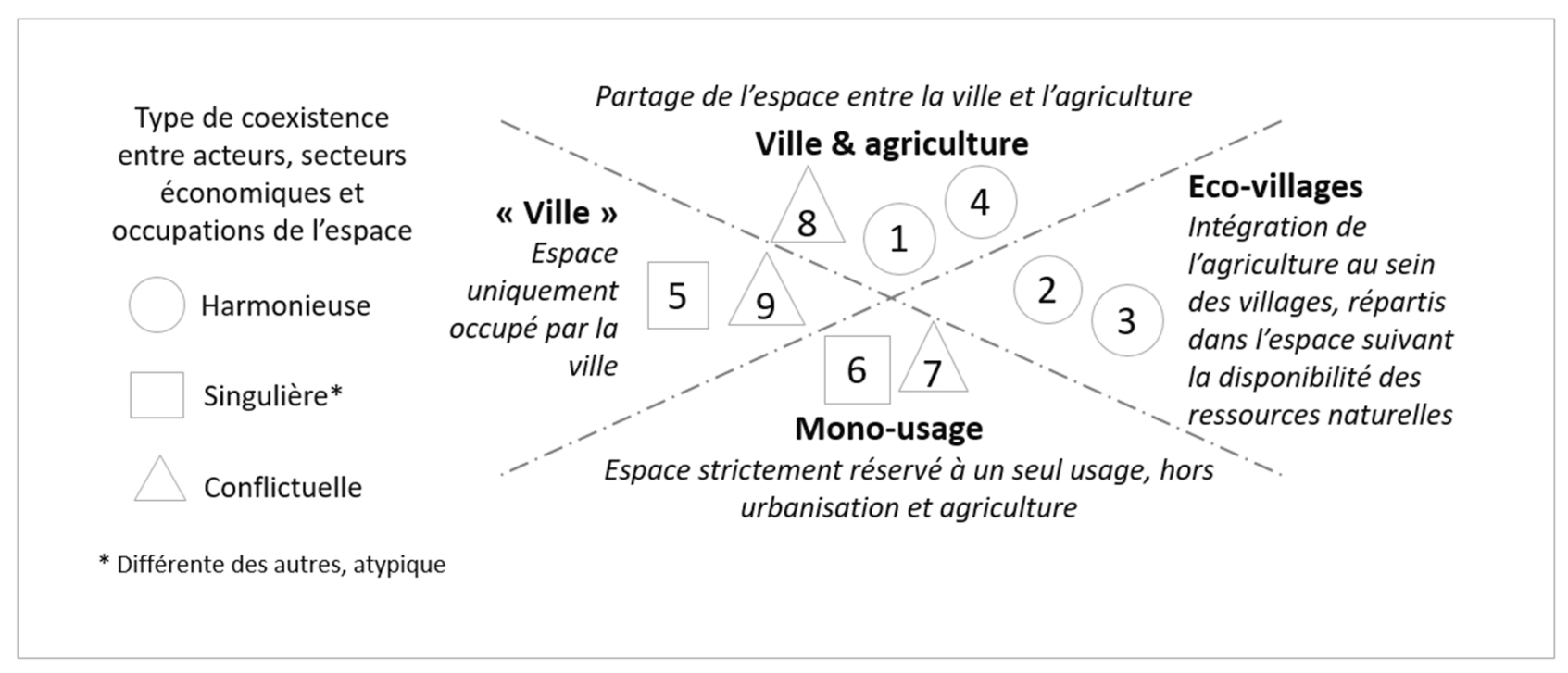

Fig. 3. Partage de l'occupation de l'espace et types de coexistence dans les neuf scénarios (se référer à la figure 2 pour les numéros des scénarios) (source: auteurs).

Fig. 3. Land use share and types of coexistence for the nine scenarios (refer to figure 2 for scenarios numbers) (source: authors).

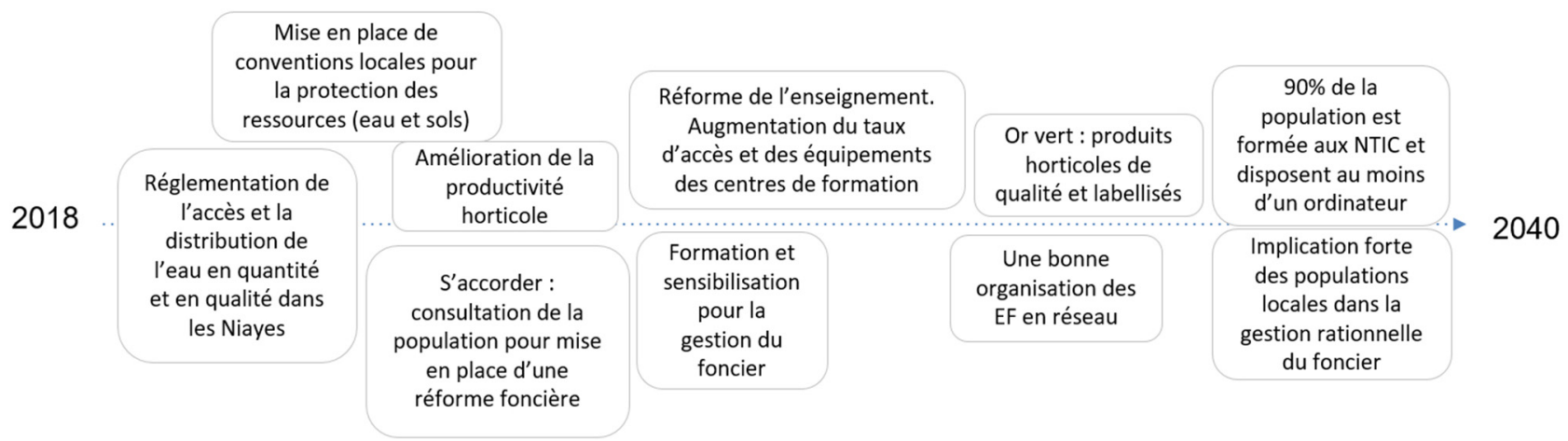

Fig. 4. Exemple des points d'inflexion pour le cheminement du scénario prospectif $\mathrm{n}^{\mathrm{o}} 1$ «L'Eldorado du Sénégal» (source: auteurs).

Fig. 4. Example of the tipping points for the prospective scenarios number 1 "a Senegalese Eldorado" (source: authors).

des réglementations sur l'accès à l'eau et sa distribution et sur l'occupation des sols, la gestion de la pression démographique et la répartition dynamique de la population, sont au cœur du bon fonctionnement de la société et du territoire dans leur ensemble. À la condition supplémentaire que les ressources naturelles soient respectées par tous et valorisées à toutes les échelles d'action ainsi que par les différentes catégories $\mathrm{d}$ 'acteurs, cette coalition mène à une gestion harmonieuse de l'espace et à la prospérité de toutes les activités mais aussi à l'amélioration de la qualité de vie des populations.

À l'opposé, la détention du pouvoir par des groupes industriels privés motivés uniquement par leur propre profit (scénarios 7, 8 et 9), aboutit à des situations conflictuelles peu avantageuses pour les populations et les activités dans la zone. Dans ces trois scénarios, soit les réglementations n'existent pas (scénario 9), soit elles ne sont pas respectées par les acteurs, du fait de l'inconscience des risques encourus ou par choix de les outrepasser, puisque l'État n'a pas les moyens de les faire respecter (scénario 8). Un dernier cas de figure est le modelage des réglementations suivant les aspirations des groupes privés, en connivence avec l'État et en relation avec la faiblesse d'action des autres acteurs du territoire (scénario 7). Dans le scénario 7, seules les ressources utiles à la réalisation de profits privés sont préservées (eau et bande de filaos); dans le scénario 9, qui peut être vu comme une aggravation du scénario 8, toutes les ressources finissent par être totalement dégradées. Dans les trois cas, les ressources utilisées par la population locale sont quant à elles dégradées.

Enfin, les scénarios 5 et 6 présentent des situations singulières. Dans le scénario 6, l'État et la population agissent de concert pour transformer la zone en une réserve naturelle à vocation touristique qui permet la préservation des ressources. Dans le scénario 5, l'État est faible mais la société civile est fortement structurée; néanmoins, de fortes inégalités se creusent au sein de la population et les ressources accessibles ne sont pas toujours de qualité. 
Une gouvernance inclusive, une société civile fortement structurée et la préservation des ressources naturelles apparaissent donc comme essentielles à l'atteinte de futurs harmonieux.

\subsection{La réflexion sur le futur, vecteur de transformation de la vision du présent}

Ce travail d'anticipation a été un défi pour les experts qui ont dû faire face à la difficulté de se détacher du présent et de ce qu'ils imaginaient pour l'avenir de leur territoire afin d'élaborer une diversité de futurs plausibles. Un premier travail réalisé au début des ateliers, où les experts explicitaient comment ils imaginaient le futur, avait ainsi mis en évidence une assez faible diversité de visions du futur, toutes plus ou moins en continuité directe avec les dynamiques du présent.

Initialement néophytes du processus de prospective, les experts sont ensuite devenus porteurs de la méthodologie ainsi que de leurs propres résultats auprès d'un public plus large. Ainsi, les experts se sont rassemblés par deux fois bénévolement pour terminer les scénarios. Un comité de diffusion des résultats obtenus a aussi été créé, rassemblant dix d'entre eux en charge de cette dynamique de diffusion, qui a conduit à ce jour à neuf réunions, trois restitutions, et la coécriture d'un film de présentation des scénarios, à diffuser à large échelle. Un planning d'organisation des restitutions auprès des populations et des décideurs est en cours d'élaboration.

Cette diffusion est portée par l'envie des experts de partager cette capacité à donner un sens différent au présent, acquise lors des ateliers, pour apprécier autrement l'urgence d'agir et les leviers à disposition pour ce faire. Ainsi, bien que les restitutions aient consisté à présenter les scénarios du futur, les débats qui ont suivi ont porté sur le présent, sur les actions à réaliser aujourd'hui et sur la similitude entre certains scénarios présentés et des dynamiques déjà observables sur le territoire. Ainsi, le scénario 7 «Zone minée» fait écho à la présence ancienne et toujours actuelle d'une industrie minière sur une importante partie du territoire, et le scénario 5 «La ville verte autogérée » a amené les participants à évoquer le pôle urbain de Diamniadio, aujourd'hui en plein essor. Le recours au futur a donc permis aux participants de ce travail d'anticipation de transformer des poches du futur dans le présent, qui sont actuellement des éléments isolés, en modèles dominants. Cela a fait naître des interrogations controversées sur le souhaitable et le non-souhaitable pour leur territoire.

À l'issue des premières restitutions, un questionnaire distribué aux 36 participants a montré que $96 \%$ d'entre eux pensent qu'il est possible d'agir dès maintenant pour aller vers un futur qu'ils considèrent souhaitable.

\section{Conclusion}

Neuf scénarios d'évolutions plausibles de l'espace agrosylvo-pastoral de la zone sud des Niayes ont été co-élaborés par un groupe d'experts locaux lors d'ateliers de prospective territoriale. Ces scénarios ont été construits à partir de 43 facteurs de changement internes ayant une influence plus ou moins directe sur les transformations du système d'étude. L'étude approfondie des point d'infléxion révèle que la gouvernance, les réglementations et la démographie sont les trois facteurs majeurs pouvant infléchir les trajectoires territoriales dans le temps. Elle souligne aussi l'importance d'une gouvernance inclusive pour une gestion durable des ressources naturelles et une coopération mutuellement bénéfique entre territoires ruraux et urbains. La nécessité d'avoir une société civile et des organisations professionnelles fortement structurées, ainsi que d'inclure la protection et la valorisation des ressources naturelles dans toutes les activités et à toutes les échelles, apparaît aussi comme essentielle.

Ce processus de construction de scénarios, en fournissant des éléments de réflexion à partir de l'exploration du futur, permet de donner un autre sens au présent (Miller, 2015) et de développer une posture pro-active (Godet et al., 2004), afin de prendre des décisions éclairées par une compréhension de leurs implications à long terme. La phase d'utilisation des scénarios qui fait suite à ce travail d'exploration a pour but, à partir de la compréhension ou de la perception du présent qu'apportent les futurs ainsi construits, de permettre d'influencer ou d'intervenir dans les décisions.

Pour cela, une phase de communication et de diffusion à grande échelle des résultats auprès des acteurs locaux est lancée. Elle vise à utiliser ce travail d'anticipation pour encourager les populations locales à porter un nouveau regard sur le présent et à participer à la conception des programmes et des projets.

Remerciements. Ce travail a été réalisé dans le cadre d'une activité de recherche menée par le Centre de coopération internationale en recherche agronomique pour le développement (CIRAD) avec l'appui du Bureau d'analyses macroéconomiques de l'Institut sénégalais de recherches agricoles (ISRA-BAME), que nous remercions. Nous remercions également le comité de pilotage, constitué du Conseil national de concertation et de coopération des ruraux (CNCR), de l'Initiative prospective agricole et rurale (IPAR) et d'Environnement et développement du Tiers-Monde-protection naturelle (ENDA PRONAT), qui soutient la démarche, ainsi que les experts qui ont co-élaboré les scénarios et qui s'impliquent activement dans la diffusion des résultats (Camara et al., 2018). Nous remercions aussi chaleureusement Jérémy Bourgoin pour son soutien et son implication durant l'organisation des ateliers. Enfin, nous remercions le Groupe de recherche et d'échange technologique (GRET) pour son investissement dans la phase de diffusion des résultats.

\section{Références}

Alex B, Gemenne F. 2016. Impacts du changement climatique sur les flux migratoires à l'horizon 2030. Observatoire des enjeux géopolitiques de la démographie, 1. France: DGRIS, 53 p.

Bispo A, Gabrielle B, Makowski D, El Akkari M, Bamière L, Barbottin A, et al. 2017. Effets environnementaux des changements d'affectation des sols liés à des réorientations agricoles, forestières ou d'échelles territoriales: une revue critique de la littérature scientifique. Paris, France: INRA-ADEME, 8 p.

Bosc PM, Losch B. 2002. Les agricultures familiales africaines face à la mondialisation : le défi d'une autre transition. $O C L$ 9: 402-408. DOI: 10.1051/ocl.2002.0402.

Bourgeois R, Liswanti N, Mukasa C, Zamora A, Herawati T, Monterroso I, et al. 2017. Guide for co-elaboration of scenarios: 
building shared understanding and joint action for reform and security of forest tenure. Bogor, Indonesia: Center for International Forestry Research (CIFOR), 70 p. DOI: 10.17528/cifor/006749.

Bricas N, Seck PA. 2004. L'alimentation des villes du Sud : les raisons de craindre et d'espérer. Cahiers Agricultures 13(1): 10-14.

Camara C, Bourgeois R, Bourgoin J, Camara A, Ciss I, Daouda GP, et al. 2018. Rapport des ateliers de co-construction de scénarios prospectifs pour la zone sud des Niayes. Dakar, Sénégal: CIRADISRA-BAME, 50 p. DOI: 10.18167/agritrop/00433.

CEDEAO. 2010. Politique industrielle commune de l'Afrique de l'Ouest - PICAO. NR: CEDEAO, $71 \mathrm{p}$.

DGPRE. 2014. Étude du plan de gestion des ressources en eau de la sous UGP Niayes (Rapport de synthèse). Dakar, Sénégal: Ministère de l'Hydraulique et de l'Assainissement, $42 \mathrm{p}$.

Fall ST, Fall AS. 2001. Cités horticoles en sursis? L'agriculture urbaine dans les grandes Niayes au Sénégal. Ottawa, Canada: CRDI, $138 \mathrm{p}$.

FAO. 2017. La situation mondiale de l'alimentation et de l'agriculture 2017. Mettre les systèmes alimentaires au service d'une transformation rurale inclusive. Rome, Italie: FAO, 178 p.

Fare Y, Dufumier M, Loloum M, Miss F, Pouye A, Khastalani A, et al. 2017. Analysis and diagnosis of the Agrarian system in the Niayes Region, Northwest Senegal (West Africa). Agriculture 7(59): 25 p. DOI: 10.3390/agriculture7070059.

Foley JA, DeFries R, Asner GP, Barford C, Bonan G, Carpenter SR, et al. 2005. Global consequences of land use. Science 309: 570574. DOI: $10.1126 /$ science.1111772.

Fourny C, Denizot D. 2007. La prospective territoriale, révélateur et outil d'une action publique territorialisée. In: Dodier R, Rouyer A, Séchet $\mathrm{R}$ (dir.). Territoires en action et dans l'action. Rennes, France: Presses universitaires de Rennes, pp. 29-44.

Godet M, Monti R, Meunier F, Roubelat F. 2004. Cahiers du LIPSOR $\mathrm{n}^{\circ} 5$-La boîte à outils de prospective stratégique. Paris, France: LIPSOR-CNAM, $114 \mathrm{p}$.

Halleux JM. 2015. Les territoires périurbains et leur développement dans le monde : un monde en voie d'urbanisation et de périurbanisation. In: Bogaert J, Halleux JM, ed. Territoires périurbains. Développement, enjeux et perspectives dans les pays $d u$ Sud. Gembloux, Belgique: Presses agronomiques de Gembloux, pp. 43-61.

Lardon S, Noucher M. 2016. Construire demain par les cartes : usages de l'information géographique en prospective territoriale participative. Cahiers de géographie du Québec 60(170): 209-219. DOI: 10.7202/1040531ar.

Loinger G, Spohr C. 2005. Prospective et planification territoriales : état des lieux et propositions. $N R$ : travaux et recherches de prospective $\mathrm{n}^{\circ} 24,198 \mathrm{p}$.

Mercandalli S, Losch B. 2018. Une Afrique rurale en mouvement. Dynamiques et facteurs des migrations au sud du Sahara. Rome, Italie: FAO-CIRAD, $60 \mathrm{p}$.

Miller R. 2015. Learning, the future, and complexity. An essay on the emergence of futures literacy. European Journal of Education 50: 513-523. DOI: 10.1111/ejed.12157.

Miller R (Ed). 2018. Transforming the future. Anticipation in the 21st century. London, England: Routledge, 300 p. DOI: 10.4324/ 9781351048002.

Mirenowicz P. 1991. Guide pour les actions et études de prospective territoriale. Paris, France: DATAR, 42 p.

Robineau O, Dugué P. 2018. A socio-geographical approach to the diversity of urban agriculture in a West African city. Landscape and Urban Planning 170: 48-58. DOI: 10.1016/j.landurb plan.2017.09.010.

Spohr C. 2009. Vers une prospective territoriale post-Grenelle de l'environnement. Questions et modes d'emploi. Paris, France: CGDD, 55 p.

Temple L, Moustier P. 2004. Les fonctions et contraintes de l'agriculture périurbaine de quelques villes africaines (Yaoundé, Cotonou, Dakar). Cahiers Agricultures 13(1): 15-22.

Wade CT. 2015. Enjeux d'un aménagement du territoire autour de la ville de Dakar: quel avenir pour l'agriculture périurbaine? AGRIDAPE, Revue sur l'agriculture durable à faibles apports externes 31: 9-12.

Citation de l'article : Camara C, Bourgeois R, Jahel C. 2019. Anticiper l'avenir des territoires agricoles en Afrique de l'Ouest: le cas des Niayes au Sénégal. Cah. Agric. 28: 12 . 